\title{
Radiation-driven Feedback to the ISM around AGNs
}

\author{
Keiichi Wada \\ Kagoshima University, Kagoshima 890-0065, Japan \\ email: wada@astrophysics.jp
}

\begin{abstract}
Although the "donut-like" obscuring molecular torus is often postulated to explain the type-1 and -2 dichotomy in AGNs, its physical origin is still unclear. We propose a plausible mechanism to explain the formation of the geometrically and optically thick torus, i.e. radiationdriven fountain. Using 3-D hydrodynamic simulations including radiative feedback from the central source, taking into account the $\mathrm{X}$-ray heating and radiation pressure on the gas, we found that a vertical circulation of gas is generated in the central few to tens parsecs. Interaction between the non-steady outflows and inflows causes the formation of a geometrically thick torus with internal turbulent motion. As a result, the AGN is obscured for a wide range of solid angles. In a quasi-steady state, the opening angles for the column density toward a black hole $<10^{23}$ $\mathrm{cm}^{-2}$ are approximately $\pm 30^{\circ}$ and $\pm 50^{\circ}$ for AGNs with $10 \%$ and $1 \%$ Eddington luminosity, respectively. Mass inflows through the torus coexist with the outflow and internal turbulent motion, although the average mass accretion rate to the central parsec region is about ten times smaller than the accretion rate required to maintain the assumed AGN luminosity. This implies that relatively luminous AGN activity is intrinsically intermittent or that there are other mechanisms, such as stellar energy feedback, that enhance the mass accretion to the center.
\end{abstract}

\section{Introduction}

Dynamical, radiative, and chemical feedback processes from AGNs to the surrounding material are crucial in terms of evolution of galaxies and supermassive black holes. Essentially the AGN feedback phenomenon covers a wide dynamic range, from micro-parsecs to mega-parsecs, but here I focus on a scale of sub-pc to tens of pc from the central engine. On this scale, the presence of optically and geometrically thick obscuring tori has been postulated. However, the real structure of the obscuring material, and how it depends on physical properties of the AGNs, remain unclear.

Although a parsec-scale dusty gas disk is observed in some nearby AGNs (e.g. Jaffe et al. 2004), the nuclei could also be obscured by relatively less dense ISM in the range of 10-100 pc or even by the galactic disks themselves (Levenson et al. 2011; Goulding et al. 2012). There are many instances where evidence suggests that most AGNs are associated with circumnuclear starbursts (Davies et al. 2007; Hick et al. 2009; Chen et al. 2009; Imanishi et al. 2011; Woo et al. 2012). It is suggested that the star formation activities drive mass inflow into AGNs, thereby leading to the growth of black holes. The nuclear starbursts themselves could inflate the circumnuclear disk and obscure the central source. Wada \& Norman (2002) and Wada et al. (2009) have proposed that a clumpy torus-like structure is naturally reproduced due to energy feedback from supernova explosions (SNe) in a disk. In their model, the internal density and temperature of the thick disk are highly inhomogeneous, and the velocity field of the disk is turbulent. The scale height of the disk is determined by the balance between the turbulent energy dissipation and energy input from SNe. 
On a parsec scale or smaller, geometrically and optically thick tori sustained by radiation pressure have been suggested (Krolik \& Begelman 1988). Following this model, static solutions of thick disks supported by infrared radiation have been explored by Shi \& Krolik (2008).

It would be more natural to assume that radiative feedback causes dynamical evolution of the surrounding ISM rather than static structures. Roth et al. (2012) calculated the net rate of momentum deposition due to the radiation pressure in the surrounding gas and estimated the mass-loss rate by outflow using Monte Carlo radiative transfer calculations. Dorodnitsyn et al. (2012) performed '2.5D' (i.e. basic equations are solved in three dimensions with axisymmetry) radiative hydrodynamic simulations, and they found that an AGN torus can be better described in terms of a radiationally supported flow rather than a quasi-static obscuring torus. Using 2D simulations, Schartmann et al.(2011) studied the evolution of dusty clouds irradiated by the AGN and found that the radiative transfer effect is significant depending on the column densities of the clouds.

In order to understand the dynamical process leading to the formation of thick tori, and to examine their structures in a quasi-steady state, we performed fully 3 -D hydrodynamic simulations on scales of a few tens of pc around an AGN. This is a natural extension of our previous three-dimensional hydrodynamic simulations (Wada \& Norman 2002; Wada \& Tomisaka 2005; Yamada et al. 2007; Wada et al. 2009).

\section{3D Radiation-Hydrodynamics: Method and Models}

Here, we solve fully 3-D hydrodynamic equations, accounting for radiative feedback processes from the central source using a ray-tracing method. We assume neither dynamical equilibrium nor geometrical symmetry. In a manner similar to the approach by Wada et al. (2002), we account for self-gravity of the gas, radiative cooling, uniform UV radiation for photoelectric heating and $\mathrm{H}_{2}$ formation/destruction (Wada et al. 2009); however, we do not include the effect of supernova feedback in this study, in order to clarify the effect of the radiation feedback only.

We use a numerical code based on Eulerian hydrodynamics with a uniform grid (Wada \& Norman 2001; Wada 2001; Wada et al. 2009) to simulate the three-dimensional evolution of a rotating gas disk in a fixed spherical gravitational potential under the influence of radiation from the central source. We use $256^{3}$ uniform grid points, covering a $64^{3} \mathrm{pc}^{3}$ region around the galactic center. The non-equilibrium chemistry of hydrogen molecules along with the hydrodynamic equations are also solved.

The radial component of the radiation pressure is: $\boldsymbol{f}_{\text {rad }}^{r}=\int \chi_{\nu} \boldsymbol{F}_{\nu}^{r} / c d \nu$, where $\chi_{\nu}$ denotes the total mass extinction coefficient due to dust absorption and Thomson scattering, i.e. $\chi_{\nu} \equiv \chi_{d u s t, \nu}+\chi_{T}$. The radial component of the flux at the radius $r, \boldsymbol{F}_{\nu}^{r}$ is $\boldsymbol{F}_{\nu}^{r} \equiv L_{\nu}(\theta) e^{-\tau_{\nu}} /\left(4 \pi r^{2}\right) \boldsymbol{e}_{r}$, where $\tau_{\nu}=\int \chi_{\nu} \rho d s$. In our study, we simply assume emission from a thin accretion disk without considering the limb darkening effect, i.e., $L_{\nu} \propto L_{A G N}|\cos \theta|$, where $\theta$ denotes the angle from the rotational axis $(z$-axis). The optical depth $\tau_{\nu}$ is calculated at every time step along a ray from the central source at each grid point, i.e. $256^{3}$ rays are used in the computational box.

The effects of hard X-rays on the thermal and chemical structures of the ISM are significant in the central tens of pc region (Pérez-Beaupuits et al. 2011). Here, we consider heating by the X-rays (Coulomb heating), and $\mathrm{H}_{2}$ ionizing heating in the warm and cold gas (Maloney et al. 1996; Meijerink \& Spaans 2005). For optically thin hot gas, the effects of Compton heating and X-ray photoionization heating are considered (Blondin 1994).

In order to prepare a quasi-steady initial condition without radiation feedback, we first run a model of an axisymmetric and rotationally supported thin disk with a uniform 


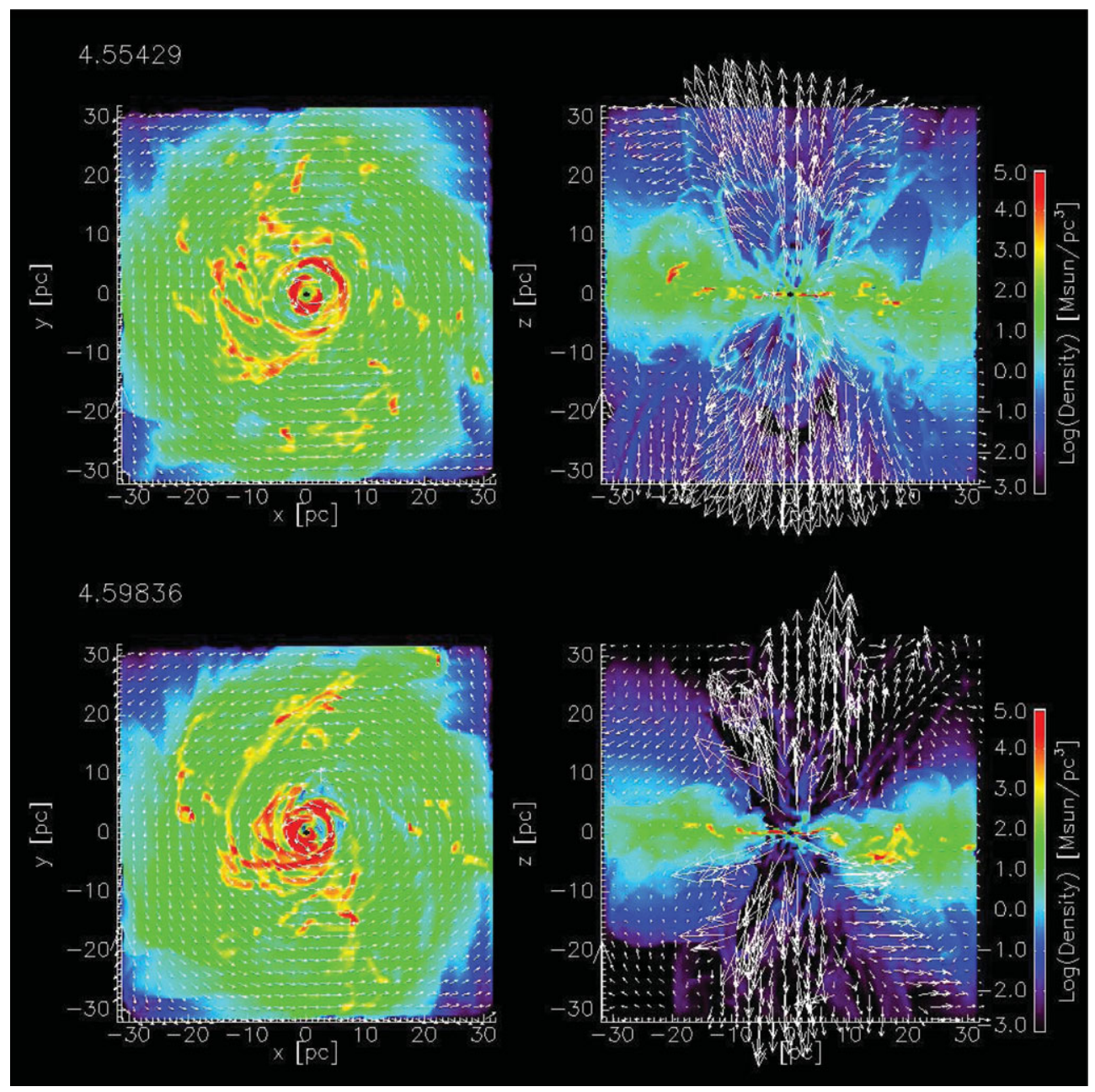

Figure 1. Gas density in quasi-steady state of two models: (top) $L_{A G N} / L_{E}=0.1$ at $t=4.55$ Myr and (bottom) $L_{A G N} / L_{E}=0.01$ at $t=4.59 \mathrm{Myr}$. The vertical slices indicate the $x$ - $z$ planes.

density profile (thickness of $2.5 \mathrm{pc}$ ) and a total gas mass of $M_{g}=6.7 \times 10^{6} M_{\odot}$. Random density fluctuations, which are less than $1 \%$ of the unperturbed values, are added to the initial gas disk.

A free parameter in the present simulations is the luminosity of the AGN. Here, we explore models with two different Eddington ratios $L_{A G N} / L_{E}=0.1$ and 0.01 , where $L_{E}$ is the Eddington luminosity.

\section{Results}

Figure 1 shows that the density structures in a quasi-steady state differ depending on the luminosity of the central source. Note that since the duration of the present simulation is much shorter than the accretion time scale $\left(\sim 10^{9} \mathrm{Myr}\right)$, 'quasi-steady' does not necessary imply long-term stability.

In the more luminous model $\left(L_{A G N} / L_{E d d}=0.1\right.$, upper panels), inhomogeneous outflows with $\rho \sim 0.1-1 M_{\odot} \mathrm{pc}^{-3}$ and velocity of $\sim 100 \mathrm{~km} \mathrm{~s}^{-1}$ are formed, and diffuse gas $\left(\rho \sim 0.1 M_{\odot} \mathrm{pc}^{-3}\right)$ extends for over $20 \mathrm{pc}$ above the equatorial plane. On the other 


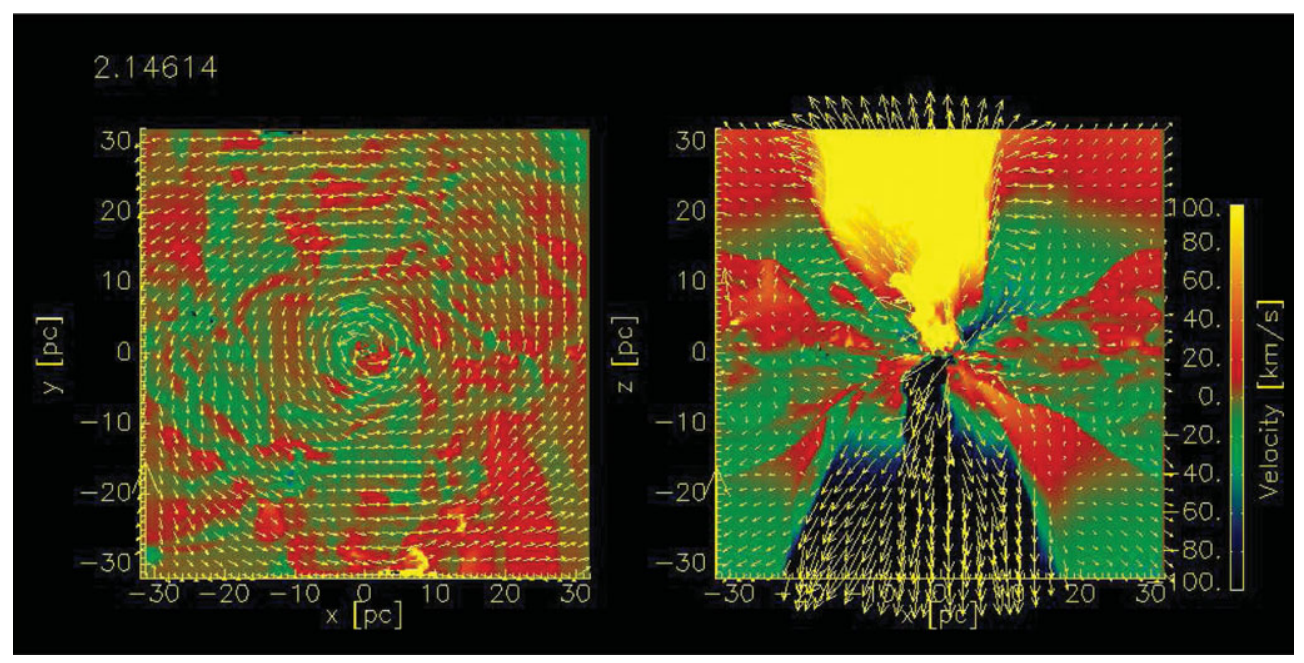

Figure 2. $z$-component of velocity $\left(L_{A G N} / L_{E}=0.1\right)$. The vertical slice indicates the $x$ - $z$ plane. The downward flows (i.e., flow heading to the equatorial plane) appear outside the region of the biconical outflow, and these flows interact with the opposite directed flows (see Fig. 3), which are represented by the regions indicated in green/blue and red/yellow. The upward and downward flows coexist and generate turbulent motions in the equatorial plane (left panel) as well as in the vertical direction.

hand, diffuse and relatively stable outflows with $\rho \sim 10^{-2} M_{\odot} \mathrm{pc}^{-3}$ or less are formed in the less luminous model with $L_{A G N} / L_{E d d}=0.01$.

We found that when biconical radiation-driven outflows are formed, "back-flows" toward the disk plane also appear outside the outflows. These accretion flows interact with the disk, thereby leading to the formation of a geometrically thick disk. The interactions of vertical flows with opposite directions are more clearly seen in Fig. $2(t=2.146 \mathrm{Myr})$, which shows the distribution of $v_{z}$. There are several contact surfaces between upward and downward flows. As a result, turbulent motions that support the thick geometrical structure of the disk are generated. The complicated distribution of flows in the thick disk is also seen in the velocity field at the equatorial plane (left panel of Fig. 2).

The downward flows can be naturally expected due to the balance between radiation pressure and the gravity. Fig. 3 schematically shows flows under the effect of radiation and gravity. Accretion flows appear in the gravity-dominated domain. Moreover, since the critical lines dividing the two domains are not purely radial, outflows with large angles from the $z$-axis may collide with the boundary of the cavity. This pushes the boundaries of the outflow outward, and the gases in the outflows change their directions and fall towards the center as seen in Fig. 3. This also causes the biconical cavities to become non-steady, and the vertical circulation of the flow, or the "fountain" of gas, circulates around the AGN on a scale of $10 \mathrm{pc}$. The kinetic energy of this circulating flow is partially used to generate the random motion in the disk and maintain its thickness. In other words, both radiation energy and gravity are driving the internal turbulent motion in the disk.

Obscuration due to the vertically extended gas in the central region over tens of parsecs and how it differs between models are shown in Fig. 4, which shows the plot of the column density toward the central $\mathrm{BH}$ as a function of the viewing angle. If there is no radiation from the central source, the opening angle for $N \leqslant 10^{23} \mathrm{~cm}^{-2}$ is approximately $140^{\circ}$. The radiation feedback causes the thickness of the disk to increase, and the opening angles are approximately $60^{\circ}$ and $100^{\circ}$ for $L_{A G N} / L_{E}=0.1$ and 0.01 , respectively. It 


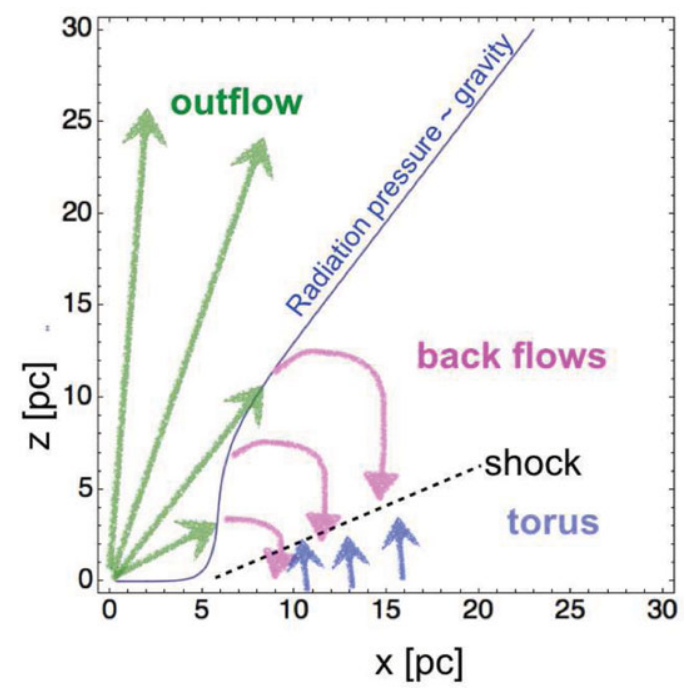

Figure 3. Radiation-driven "fountain" in the central tens pc under the effects of the central radiation and gravity. The solid line is a critical line between radiation-pressure dominant and gravity dominant regions for a gas disk. Note that the structure is not static, but highly dynamic.
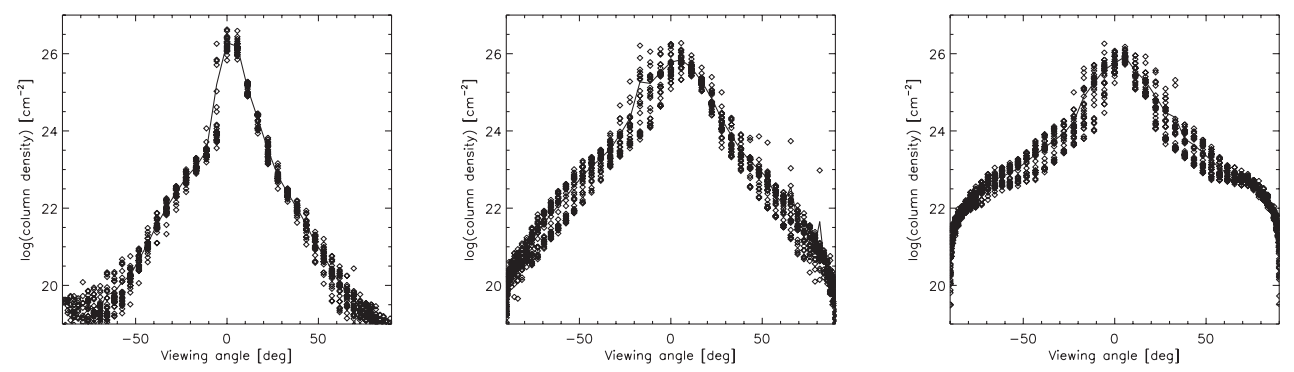

Figure 4. Total column density distribution as a function of viewing angles $\left(90^{\circ}\right.$ : pole-on view and $0^{\circ}$ : edge-on view) and azimuthal angles. For each viewing angle, the column density is plotted for 50 azimuthal angles. The solid line indicates the azimuthal averaged column density for the viewing angles. (left) Before the radiation feedback is turned on, (middle) $L_{A G N} / L_{E}=0.01$, (right) $L_{A G N} / L_{E}=0.1$.

is noteworthy that the column density does not only depend on the viewing angle, but also on the azimuthal angles. The column density varies by a factor of ten or more for a given viewing angle, thereby indicating the internal inhomogeneous structure of the disk. The structure of the disk is similar to that generated by supernova explosions (Wada \& Norman 2002, Wada et al. 2009). However, the present simulations imply that the geometrically and optically thick torus can be naturally formed due to gravitational energy in the central part with the aid of the radiation feedback.

The radiation pressure caused by the central source usually has a "negative" effect on the mass accretion toward the center; however, our results indicate that mass accretion is not terminated by radiative feedback. We found that the lower luminosity model $\left(L_{A G N} / L_{E d d}=0.01\right)$ shows continuous net mass accretion in the central 8-pc region, and there is almost no net inflow beyond $r \sim 4 \mathrm{pc}$. The mass accretion in the central part is caused by gravitational torque due to the non-axisymmetric features near the equatorial plane of the central part of the disk. The accretion rate to the central parsec increases with decreasing AGN luminosity: $\dot{M}=1.7 \times 10^{-3} M_{\odot} \mathrm{yr}^{-1}$ and $\dot{M}=1.3 \times 10^{-4} M_{\odot} \mathrm{yr}^{-1}$ 
for $L_{A G N} / L_{E}=0.01$ and 0.1 , respectively. For $L_{A G N}=0.1 L_{E}$, the net accretion rate is one order of magnitude smaller than the accretion rate required to maintain constant AGN luminosity; the AGN luminosity is assumed to be constant during the calculations provided that the energy conversion efficiency is 0.1 . On the other hand, the observed accretion rate in the low luminosity AGN model is comparable to that required for the luminosity of the source. These results may suggest that luminous AGNs are intermittent, and their luminosities are not instantaneously determined by mass inflow from $r \sim 1 \mathrm{pc}$.

\section{References}

Alexander, D. M. \& Hickox, R. C. 2011, arXiv:1112.1949

Blondin, J. M. 1994, ApJ, 435, 756

Chen, Y.-M., Wang, J.-M., Yan, C.-S., Hu, C., \& Zhang, S. 2009, ApJl, 695, L130

Dorodnitsyn, A., Kallman, T., \& Bisnovatyi-Kogan, G. S. 2012, ApJ, 747, 8

Diamond-Stanic, A. M. \& Rieke, G. H. 2012, ApJ, 746, 168

Dalgarno, A., Yan, M., \& Liu, W. 1999, ApJs, 125, 237

Davies, R. I., Sánchez, F. M., Genzel, R., Tacconi, L. J., Hicks, E. K. S., Friedrich, S., \& Sternberg, A. 2007, ApJ, 671, 1388

Goulding, A. D., Alexander, D. M., Bauer, F. E., et al. 2012, arXiv:1205.1800

Hambrick, D. C., Ostriker, J. P., Naab, T., \& Johansson, P. H. 2011, ApJ, 738, 16

Hicks, E. K. S., Davies, R. I., Malkan, M. A., Genzel, R., Tacconi, L. J., Sánchez, F. M., \& Sternberg, A. 2009, ApJ, 696, 448

Imanishi, M., Ichikawa, K., Takeuchi, T., et al. 2011, PASJ, 63, 447

Imanishi, M. \& Wada, K. 2004, ApJ, 617, 214

Jaffe, W., et al. 2004, Nature, 429, 47

Krolik, J. H. 2007, ApJ, 661, 52

Krolik, J. H. \& Begelman, M. C. 1988, ApJ, 329, 702

Levenson, N. A., Weaver, K. A., \& Heckman, T. M. 2001, ApJs, 133, 269

Maloney, P. R., Hollenbach, D. J., \& Tielens, A. G. G. M. 1996, ApJ, 466, 561

Meijerink, R. \& Spaans, M. 2005, A\&Ap, 436, 397

Pérez-Beaupuits, J. P., Wada, K., \& Spaans, M. 2011, ApJ, 730, 48

Roth, N., Kasen, D., Hopkins, P. F., \& Quataert, E. 2012, arXiv:1204.0063

Shi, J. \& Krolik, J. H. 2008, ApJ, 679, 1018

Schartmann, M., Krause, M., \& Burkert, A. 2011, MNRAS, 415, 741

Wada, K. 2001, ApJl, 559, L41

Wada, K., Meurer, G., \& Norman, C. A. 2002, ApJ, 577, 197

Wada, K. \& Norman, C. A. 2001, ApJ, 547, 172

Wada, K. \& Norman, C. A. 2002, ApJl, 566, L21 (WN02)

Wada, K. \& Tomisaka, K. 2005, ApJ, 619, 93

Wada, K., Papadopoulos, P. P., \& Spaans, M. 2009, ApJ, 702, 63

Woo, J.-H., Kim, J. H., Imanishi, M., \& Park, D. 2012, AJ, 143, 49

Yamada, M., Wada, K., \& Tomisaka, K. 2007, ApJ, 671, 73 